\title{
Electron Transport and Spin Scattering in Very Thin Disordered Metallic Films
}

\begin{abstract}
A. PAJA* AND B.J. SPISAK
Faculty of Physics and Applied Computer Science

AGH University of Science and Technology, 30-059 Kraków, Poland

We consider the electron transport through a very thin disordered metallic film doped with magnetic impurities. We treat the film as a quasi-two-dimensional system with structural disorder where some ions have spins and other are spinless. The interaction of conduction electrons with localized spins is described by means of the exchange term of the Hamiltonian. The scattering is treated in the first Born approximation and the potential is assumed to be the Coulomb screened one. The total effective cross-section is calculated as a sum of the part responsible for the potential scattering and the second part which comes from the spin-spin scattering. The Fermi sphere splits into separate sheets due to the finite size of the system in the $z$ direction, therefore, the cross-section and the relaxation time are calculated for each sheet independently. The total transport relaxation time and the conductivity are obtained as functions of the thickness of the system and the contents of magnetic impurities. Some model calculations have been made for a thin disordered film of copper doped with manganese.
\end{abstract}

PACS numbers: 72.15.Cz, 72.25.Ba, 73.50.-h, 73.63.--b

\section{Introduction}

The theoretical description of the electron transport through various nanostructures is very important for further development of nanoelectronics [1]. The electrons carry not only the charge but also the spin and this fact is widely exploited in spintronics [2-4]. It should certainly be taken into account when some element of a nanocircuit contains magnetic impurities because an additional scattering then appears [5-7]. The experimental results have stimulated the development of different approaches to the problem of transport in such structures [8-10]. In this work we concentrate on the problem of conduction of the polarized carriers through a very thin disordered metallic film doped with magnetic impurities thus forming a kind of a binary alloy. A novelty in our approach is that we take into account correlations in locations of potentials by means of structure factors, contrary to other authors which use uncorrelated delta-like potentials. We treat

*corresponding author; e-mail: paja@agh.edu.pl 
the electron gas as quasi-two-dimensional due to a finite size of the system in the $z$ direction. The Fermi sphere then splits into separate sheets, as was proved in our previous work [11] and independently by Balcerzak [12]. Thus we first calculate the transport relaxation time for electrons in one specific sheet of the Fermi surface and then sum up over all sheets to get the total conductivity as a function of the thickness of thin film and the concentration of magnetic impurities. This is essential for producing spintronic nanodevices having specifically determined properties.

\section{Theoretical model}

Let us consider thin disordered metallic film, which contains $N_{A}$ nonmagnetic atoms and $N_{B}$ magnetic impurities positioned at random. Let us assume that the impurities concentration $x=N_{B} /\left(N_{A}+N_{B}\right)$ is small and they are polarized along the $z$ axis, perpendicular to the film. Because of the finite size $L_{z}$ of the film the Fermi sphere splits into a finite number of sheets lying in the planes perpendicular to the $z$ axis $[11,12]$. We treat the set of electrons which belong to such a sheet as an independent subband of constant $k_{z}$. We describe their coordinates in real and reciprocal spaces within these sheets by the vectors $\boldsymbol{\rho}$ and $\boldsymbol{k} \boldsymbol{\rho}$, respectively.

The dynamics of the electron in such film is given by the Hamiltonian

$$
\hat{H}=-\frac{\hbar^{2}}{2 m^{*}} \nabla^{2}+U(\boldsymbol{\rho}),
$$

where $m^{*}$ is the electron effective mass. The potential $U(\boldsymbol{\rho})$ is a sum of two terms

$$
U(\boldsymbol{\rho})=U_{0}(\boldsymbol{\rho})+U_{s s}(\boldsymbol{\rho}),
$$

where $U_{0}(\boldsymbol{\rho})=\sum_{l=1}^{N_{A}} u\left(\boldsymbol{\rho}-\boldsymbol{R}_{l}\right)+\sum_{i=1}^{N_{B}} u\left(\boldsymbol{\rho}-\boldsymbol{R}_{i}\right)$ is the potential of the system $\left(u\left(\boldsymbol{\rho}-\boldsymbol{R}_{l}\right)\right.$ and $u\left(\boldsymbol{\rho}-\boldsymbol{R}_{i}\right)$ are the potentials of the individual ions located at the respective sites $\boldsymbol{R}_{l}$ or $\left.\boldsymbol{R}_{i}\right)$. $U_{s s}(\boldsymbol{\rho})$ represents energy of the interaction of the conduction electron spin, $s(\boldsymbol{\rho})$, with the spins of magnetic impurities localized at the sites $\boldsymbol{R}_{i}$. It can be expressed as [13]

$$
U_{s s}(\boldsymbol{\rho})=-\sum_{i=1}^{N_{B}} J_{0}\left(\boldsymbol{\rho}-\boldsymbol{R}_{i}\right) \boldsymbol{S}\left(\boldsymbol{R}_{i}\right) \cdot \boldsymbol{s}(\boldsymbol{\rho}),
$$

where $\boldsymbol{S}\left(\boldsymbol{R}_{i}\right)$ is the impurity spin operator.

We further assume that the coupling constant of the interaction between the conduction electron and the magnetic impurity has a form typical for the contact interaction

$$
J_{0}\left(\boldsymbol{\rho}-\boldsymbol{R}_{i}\right)=J_{0} \delta\left(\boldsymbol{\rho}-\boldsymbol{R}_{i}\right)
$$

and then Eq. (3) takes the form

$$
U_{s s}(\boldsymbol{\rho})=-J_{0} \sum_{i=1}^{N_{B}} S_{z}\left(\boldsymbol{R}_{i}\right) s_{z}(\boldsymbol{\rho}) \delta\left(\boldsymbol{\rho}-\boldsymbol{R}_{i}\right),
$$

which comes from our primary assumption of complete polarization of the impurities. 
The problem of scattering of conduction electrons in such system can be conveniently described by means of the Lipmann-Schwinger integral equation [14]

$$
\Psi(\boldsymbol{\rho})=\Phi(\boldsymbol{\rho})+\frac{2 m^{*}}{\hbar^{2}} \int \mathrm{d}^{2} \rho^{\prime} G\left(\boldsymbol{\rho}, \boldsymbol{\rho}^{\prime}\right) U\left(\boldsymbol{\rho}^{\prime}\right) \Psi\left(\boldsymbol{\rho}^{\prime}\right),
$$

where $\Psi(\boldsymbol{\rho})$ and $\Phi(\boldsymbol{\rho})$ are spinors, whereas $G\left(\boldsymbol{\rho}, \boldsymbol{\rho}^{\prime}\right)$ is the Green function given by

$$
G\left(\boldsymbol{\rho}, \boldsymbol{\rho}^{\prime}\right)=-\frac{1}{(2 \pi)^{2}} \int \mathrm{d}^{2} q \frac{\exp \left(\mathrm{i} \boldsymbol{q} \cdot\left(\boldsymbol{\rho}-\boldsymbol{\rho}^{\prime}\right)\right)}{q^{2}-k_{\rho}^{2}},
$$

where $k_{\rho}$ is the maximal value of the wave vector in the $x-y$ plane for a specific sheet.

We apply the perturbation method to solve the integral equation (6) which allows us to find the differential cross-section in the first Born approximation [15]. The use of this method means that we focus on the case of weak electron-impurity scattering specified by the Ioffe-Regel criterion [16]. In this case the weak localization corrections which are caused by quantum interference effects are small compared with the Drude conductivity [17-19]. The total effective differential cross-section for scattering of the completely polarized conduction electrons in the considered system has the following form:

$$
\frac{\mathrm{d} \sigma}{\mathrm{d} \varphi}=\frac{\mathrm{d} \sigma_{0}^{(A)}}{\mathrm{d} \varphi}+\frac{\mathrm{d} \sigma_{0}^{(B)}}{\mathrm{d} \varphi}+\frac{\mathrm{d} \sigma_{\uparrow \uparrow}^{(B)}}{\mathrm{d} \varphi}
$$

where the upper indices, $A$ and $B$, denote the atoms of the matrix and impurities, respectively. The lower index 0 marks the cross-sections related to the potential scattering while index $\uparrow \uparrow$ marks the one responsible for the spin-spin scattering.

The effective cross-section for polarized electrons was calculated in [11] as

$$
\frac{\mathrm{d} \sigma_{0}^{(i)}}{\mathrm{d} \varphi}=\frac{1}{8 \pi k_{\rho}}\left(\frac{m^{*}}{2 \pi \hbar^{2}}\right)^{2} N_{i} S_{i}(q)\left|u_{a_{i}}(q)\right|^{2},
$$

where $u_{a_{i}}$ is the Fourier transform of the $i$-th type of atomic scattering potential, $S_{i}(q)$ is the $i$-th partial structure factor, and $N_{i}$ is the number of $i$-th type of atoms in the system. Using the partial structure factors in the calculations means that we assume a realistic case that scattering potentials have a finite range, comparable with the size of atoms, contrary to other authors who treat the scattering potentials as delta-like ones. The values of the wave vectors $k_{\rho}$ and $q$ have the form [11]

$$
\begin{aligned}
& k_{\rho}=\sqrt{\frac{2 m^{*}}{\hbar^{2}} E_{\mathrm{F}}-\left(\frac{m \pi}{L_{z}}\right)^{2}}, \\
& q=2 k_{\rho} \sin (\varphi / 2),
\end{aligned}
$$

where $m$ is the subband index and $L_{z}$ - the thickness of the film.

The formula for effective differential cross-section given by Eq. (8) is an approximation because we neglected the cross terms coming from the fact that the considered system is a two-component one. A numerical analysis has shown 
that the contribution of the neglected term which describes the correlations between different atoms $(A-B)$ is approximately constant and its value is small in comparison to the correlations between atoms of the same type $(A-A, B-B)$.

The effective cross-section for the scattering of electrons from the impurity spins has the form

$$
\frac{\mathrm{d} \sigma_{\uparrow \uparrow}^{(B)}}{\mathrm{d} \varphi}=\frac{1}{8 \pi k_{\rho}}\left(\frac{m^{*} J_{0}}{2 \pi \hbar^{2}}\right)^{2} S(S+1) N_{B} S_{B}(q) .
$$

This completes the formula (8) and makes it ready for calculations.

\section{Transport relaxation time}

A general formula for the inverse transport relaxation time in the quasi-two-dimensional system has the form [11]

$$
\tau^{-1}=2 \frac{v_{\mathrm{F}}}{A} \int_{0}^{\pi} \mathrm{d} \varphi \frac{\mathrm{d} \sigma}{\mathrm{d} \varphi}(1-\cos \varphi),
$$

where $A$ is the area of its surface. We now have to assume something about the structure factors and atomic potentials to proceed further. The considered system is actually a two-component amorphous alloy. Its structure should be characterized by three structure factors, in principle. However, if the concentration of one component is small then quite an accurate description of the structure can be done by only two partial structure factors, which we denote $S_{A}(q)$ and $S_{B}(q)$, respectively. To describe the transport properties of thin film we can approximate both structure factors by power functions which is a good approximation at least for $q<2 k_{\mathrm{F}}$. We found that the best approximation is $S_{A}(q)=\alpha_{A} q^{6}$ and $S_{B}(q)=$ $\alpha_{B} q^{6}$.

Atomic potential can be described by means of the Coulomb screened potential

$$
u_{a_{i}}(\rho)=u_{i} \frac{\exp \left(-\lambda_{i} \rho\right)}{\rho},
$$

where $\lambda_{i}$ is the inverse screening length in the Thomas-Fermi model. The Fourier transform of the potential (14) has the following form:

$$
u_{a_{i}}(q)=2 \pi u_{i} \frac{1}{\sqrt{\lambda_{i}^{2}+q^{2}}} .
$$

Substituting all that to Eq. (13) we obtain the inverse total transport relaxation time as the sum of three terms

$$
\tau^{-1}=\tau_{A}^{-1}+\tau_{B}^{-1}+\tau_{\uparrow \uparrow}^{-1}
$$

where the inverse transport relaxation time $\tau_{A}^{-1}$ has the form

$$
\begin{aligned}
\tau_{A}^{-1} & =\frac{8}{\pi} \frac{m^{*}}{\hbar^{3}}(1-x) n \alpha_{A} k_{\rho}^{4} u_{A}^{2} \int_{0}^{\pi} \mathrm{d} \varphi \frac{\sin ^{8}(\varphi / 2)}{\left(\frac{\lambda_{A}}{2 k_{\rho}}\right)^{2}+\sin ^{2}(\varphi / 2)} \\
& \equiv \frac{8}{\pi} \frac{m^{*}}{\hbar^{3}}(1-x) n \alpha_{A} k_{\rho}^{4} u_{A}^{2} I\left(\frac{\lambda_{A}}{2 k_{\rho}}\right)
\end{aligned}
$$


and, analogously, $\tau_{B}^{-1}$ has the form

$$
\begin{aligned}
\tau_{B}^{-1} & =\frac{8}{\pi} \frac{m^{*}}{\hbar^{3}} x n \alpha_{B} k_{\rho}^{4} u_{B}^{2} \int_{0}^{\pi} \mathrm{d} \varphi \frac{\sin ^{8}(\varphi / 2)}{\left(\frac{\lambda_{B}}{2 k_{\rho}}\right)^{2}+\sin ^{2}(\varphi / 2)} \\
& \equiv \frac{8}{\pi} \frac{m^{*}}{\hbar^{3}} x n \alpha_{B} k_{\rho}^{4} u_{B}^{2} I\left(\frac{\lambda_{B}}{2 k_{\rho}}\right)
\end{aligned}
$$

whereas the inverse spin-spin transport relaxation time $\tau_{\uparrow \uparrow}^{-1}$ can be expressed as

$$
\tau_{\uparrow \uparrow}^{-1}=\frac{8}{\pi} \frac{m^{*}}{\hbar^{3}} x n \alpha_{B} k_{\rho}^{6} J_{0}^{2} S(S+1) \int \mathrm{d} \varphi \sin ^{8}(\varphi / 2),
$$

where $n=N / A$ denotes the average area density of atoms.

After inserting the formulae (17)-(19) into Eq. (16) we obtain the inverse transport relaxation time for the $m$-th sheet of the Fermi surface

$$
\begin{aligned}
\tau_{m}^{-1} & =\frac{8}{\pi} \frac{m^{*}}{\hbar^{3}} n\left\{(1-x) \alpha_{A} k_{\rho}^{4} u_{A}^{2} I\left(\frac{\lambda_{A}}{2 k_{\rho}}\right)\right. \\
& \left.+x \alpha_{B} k_{\rho}^{4}\left[u_{B}^{2} I\left(\frac{\lambda_{B}}{2 k_{\rho}}\right)+\frac{35 \pi}{128} k_{\rho}^{2} J_{0}^{2} S(S+1)\right]\right\}
\end{aligned}
$$

where the dependence on the number $m$ comes from $k_{\rho}$ (Eq. (10)). The total conductivity of the film is given by the sum of the partial conductivities for each sheet

$$
\sigma\left(L_{z}, x\right)=\sum_{m=0}^{m_{\max }} \sigma_{m}\left(L_{z}, x\right),
$$

where the conductivity for $m$-th sheet

$$
\sigma_{m}\left(L_{z}, x\right)=\frac{1}{L_{z}} \frac{n_{m} e^{2} \tau_{m}}{m^{*}}
$$

depends on the area concentration of carriers, $n_{m}$, in each sheet, either [20]. The upper limit of the summation, $n_{\max }$, depends on the system size $L_{z}$.

We analyzed, as an example, a specific system which consists of the matrix made of copper (component $A$ ) with manganese impurities (component $B$ ). We evaluated the total electrical conductivity $\sigma$ as a function of the manganese concentration $x$ for various thicknesses $L_{z}$ of the film. The results are shown in Fig. 1. The conductivity slightly rises with a concentration of magnetic impurities. It can be understood because we assumed polarized subsystem of magnetic ions and polarized current of carriers. Their polarizations are compatible which makes the transport easier. The absolute values of conductivity decrease with the thickness $L_{z}$ of the film. It is an intrinsic feature of the model, because $\sigma$ is - roughly inversely proportional to $L_{z}^{2}$.

\section{Conclusions}

We have shown how to calculate the conductivity of a very thin disordered metallic film with magnetic impurities when the electric current consists of fully polarized electrons. The final results depend on the thickness of the film and the 


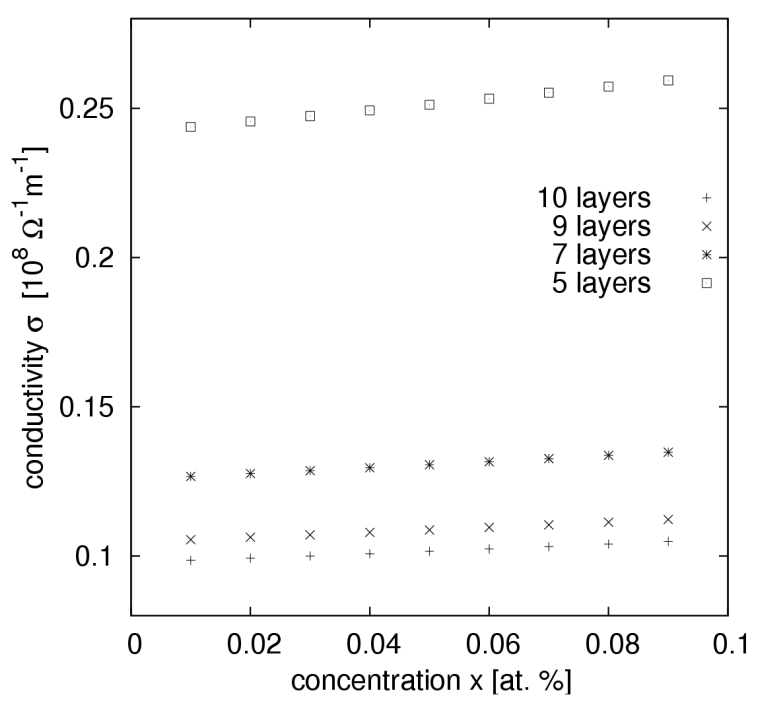

Fig. 1. The electrical conductivity $\sigma$ of a very thin disordered metallic film with magnetic impurities as a function of the concentration $x$ of these impurities for several values of the film thickness $L_{z}$.

concentration of the impurities. The conductivity increases with concentration of magnetic impurities and decreases with the thickness of the film. These results can be important for constructing some spintronics devices.

\section{Acknowledgments}

The work has been done within the framework of the statutory research of the Faculty of Physics and Applied Computer Science, AGH University of Science and Technology.

\section{References}

[1] M.K. Weldon, K.T. Queeney, J. Eng, K. Raghavachari, Y.J. Chabal, Surf. Sci. 500, 859 (2002).

[2] G. Prinz, Phys. Today 48, 58 (1995).

[3] G. Reiss, H. Brückl, A. Hütten, J. Schmalhorst, M. Justus, A. Thomas, S. Heitmann, Phys. Status Solidi B 236, 289 (2003).

[4] I. Zutic, J. Fabian, S. Das Sarma, Rev. Mod. Phys. 76, 323 (2004).

[5] J. Fabian, S. Das Sarma, J. Vac. Sci. Technol. B 17, 1708 (1999).

[6] D. Fedorov, P. Zahn, I. Mertig, Thin Solid Films 473, 346 (2005).

[7] A. Kaminski, L.I. Glazman, Phys. Rev. Lett. 86, 2400 (2001).

[8] L.M. Hernandez, A. Bhattacharya, K.A. Parendo, A.M. Goldman, Phys. Rev. Lett. 91, 126801 (2003).

[9] O. Pfennigstorf, A. Petkova, Z. Kallassy, M. Henzlera, Eur. Phys. J. B 30, 111 (2002). 
[10] N. Giordano, M.A. Pennington, Phys. Rev. B 45, 14238 (1992).

[11] B.J. Spisak, A. Paja, Mol. Phys. Rep. 40, 144 (2004).

[12] T. Balcerzak, Thin Solid Films 500, 341 (2006).

[13] C. Hass, Phys. Rev. 168, 531 (1968).

[14] S.K. Adhikari, Am. J. Phys. 54, 362 (1986).

[15] I. Bialynicki-Birula, M. Cieplak, J. Kaminski, Theory of Quanta, Oxford University Press, New York 1992.

[16] M.R. Graham, C.J. Adkins, H. Behar, R. Rosenbaum, J. Phys., Condens. Matter 10, 809 (1998)

[17] I. Aleiner, B.L. Altshuler, M.E. Gershenson, Waves Random Media 9, 201 (1999).

[18] B. Kramer, A. MacKinnon, Rep. Prog. Phys. 56, 1469 (1993).

[19] G. Bergmann, Phys. Rep. 107, 1 (1984).

[20] B.J. Spisak, A. Paja, Mater. Sci. (Poland) 24, 605 (2006). 